\title{
New Method for Determination of Free-Radical Production Efficiency of Organic Peroxides and Azo Compounds
}

\author{
Yasumasa Watanabe, ${ }^{\dagger}$ Hideyo Ishigaki, Hiroshi Okada, and Shuji Suyama
}

Fine Chemicals \& Polymers Research Laboratory, NOF Corporation,

Taketoyo-cho, Chita-gun, Aichi 470-23, Japan

(Received February 24, 1997)

\begin{abstract}
Determination of free-radical production efficiency of $t$-butylperoxy 2-ethylhexanoate (BuO) and $2,2^{\prime}$-azobisisobutyronitrile (AIBN) has been studied in $n$-alkanes using 2,4-diphenyl-4-methyl-1-pentene ( $\alpha$-methylstyrene dimer, MSD) as a model compound of styrene and as a radical trapping agent. The efficiency of BuO was higher than that of AIBN by $0.1-0.2$. Both efficiencies were decreased with carbon number (viscosity) of $n$-alkanes. The efficiency of AIBN determined agreed very well with that reported in the mixture of styrene $/ n$-hexane when compared at the same medium viscosity. MSD trapping is useful for predicting initiator efficiency of organic peroxides and azo compounds in styrene polymerization.

KEY WORDS Radical Trapping/Initiator Efficiency / Addition-Fragmentation / 2,2'-Azobisisobutyronitrile / Peroxyester / $\alpha$-Methylstyrene Dimer /
\end{abstract}

Most commercial vinyl polymers such as polystyrene and poly(methyl methacrylate) are produced by free radical polymerization, where organic peroxides and azo compounds are used as free radical initiators. These initiators decompose thermally by homolytic cleavage to give free radicals which initiate the polymerization of vinyl monomers. Thus, initiator fragments are incorporated into polymer structure as end groups. Since polymer end groups have profound influence on polymer properties such as thermal and light stability, it is quite important to clarify the initiation mechanisms of organic peroxides and azo compounds, in particular, for commercially useful initiators.

Recently, we developed a new method for investigating the initiation mechanisms of organic peroxides using 2,4-diphenyl-4-methyl-1-pentene ( $\alpha$-methylstyrene dimer, MSD) as a radical trapping agent. ${ }^{1}$ MSD trapping is based on the unique radical chemistry of MSD, that is, addition-fragmentation reaction between free radicals and MSD (eq 1 and 2). ${ }^{2}$ The easy deactivation of cumyl radicals by self-reactions (eq 3 ) also contributes to this success.
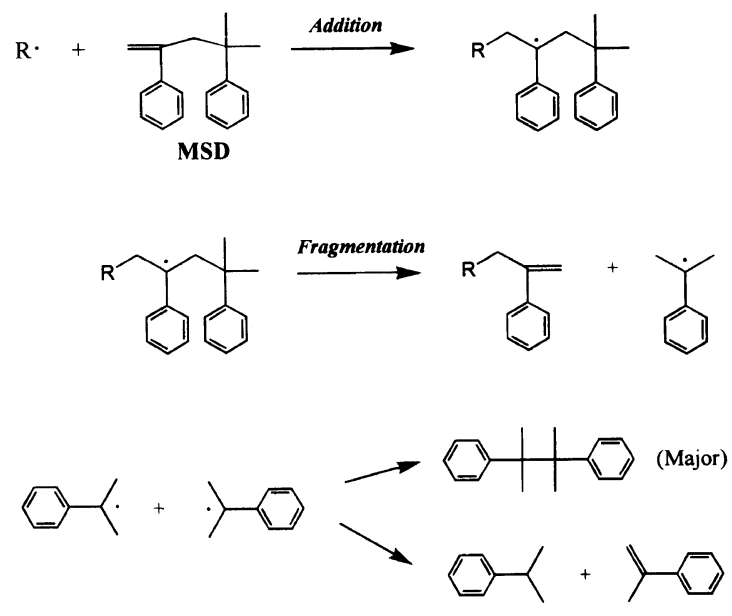

\footnotetext{
$\uparrow^{\dagger}$ To whom all correspondence should be addressed.
}

The outline of MSD trapping technique is depicted in Scheme 1. When organic peroxides decompose in MSD, stable olefinic compounds (1) having various fragments (X) derived from organic peroxides are produced through addition-fragmentation reaction. By analyzing these trapping products (1), we obtain useful information on

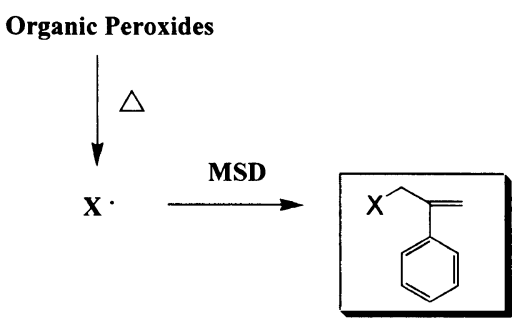

(1)

Scheme 1. Outline of MSD trapping.

the free radical chemistry of organic peroxides. We demonstrated that the double bond reactivity of MSD is about the same as that of styrene. ${ }^{1}$ Therefore, the trapping products are closely related to initiating radicals in styrene polymerization.

Some organic peroxides ${ }^{3-8}$ and azo compounds ${ }^{8-12}$ undergo cage reactions during decomposition, resulting in reduction in free-radical production efficiency (proportion of radicals diffusing out of cage). This means that initiator efficiency (proportion of radicals initiating polymerization) of peroxides and azo compounds is not always unity and changes with the environment. Since initiator efficiency greatly affects polymerization rates and polymer molecular weights, an understanding of initiator efficiency is quite important for polymer producers.

A previous paper ${ }^{13}$ reported the initiation mechanism of $t$-butylperoxy laurate (BuL) using MSD trapping, and showed that decarboxylation of lauroyloxy radicals are too fast to be trapped by MSD and the free-radical production efficiency of BuL is significantly reduced by cage reactions. Cage reactions of aliphatic peresters occur between alkyl radicals produced after decarboxylation of acyloxy radicals and alkoxy radicals (Scheme 2). This means that the number of free alkyl radicals is always 


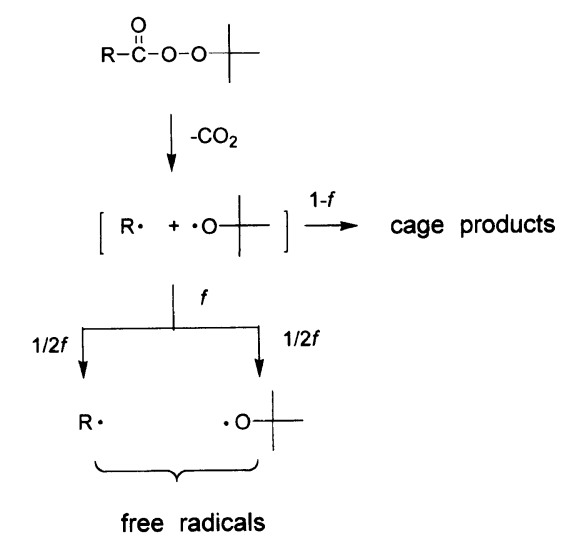

Scheme 2. Decomposition of aliphatic peresters.

equal to that of free alkoxy radicals. While the complete trapping of free alkoxy radicals by MSD was difficult due to occurrence of a side reaction (hydrogen abstraction), alkyl radicals were trapped by MSD quantitatively. This suggests that free-radical production efficiency of aliphatic peresters can be easily determined from the trapping yield of alkyl radicals produced after decarboxylation of acyloxy radicals.

2,2'-Azobisisbutyronitrile (AIBN) decomposes to give 1-cyano-1-methylethyl radicals. ${ }^{8-10}$ The alkyl radicals are also expected to undergo selective addition to MSD. Therefore, the radical efficiency of AIBN should be determined by MSD as well.

$t$-Butylperoxy 2-ethylhexanoate $(\mathrm{BuO})$ and AIBN are commercially useful initiators and their thermal stability (decomposition temperature) is quite similar. It is interesting to compare their free-radical production efficiency. This paper describes the determination of free-radical production efficiency of $\mathrm{BuO}$ and AIBN in $\mathrm{MSD} / n$-alkanes using MSD trapping. The results clearly show that $\mathrm{BuO}$ is a more efficient initiator than AIBN.

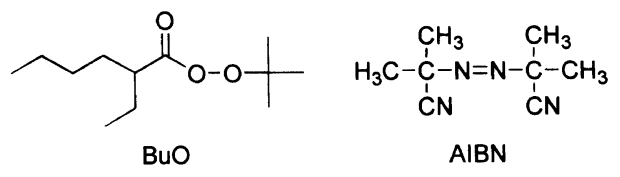

\section{EXPERIMENTAL}

\section{Measurements}

GLC analyses were performed with Shimadzu GC-14A and GC-17A gas chromatographs with a flame ionization detector using capillary columns $(15 \mathrm{~m} \times 0.53 \mathrm{~mm}$ or $25 \mathrm{~m} \times 0.25 \mathrm{~mm}$ ). GC-MS analyses were conducted on a Shimadzu QP5000 under electron impact conditions $(70 \mathrm{eV})$

\section{Materials}

2,4-Diphenyl-4-methyl-1-pentene (MSD) was obtained from NOF Corporation (Nofmer MSD) and used after purifying by distillation. BuO was obtained from NOF Corporation (perbutyl O, 99.3\% purity). AIBN and $n$-alkanes were commercially available (guaranteed reagent grade).

\section{MSD Trapping Experiments}

An aliquot $(2 \mathrm{ml})$ of $0.02 \mathrm{M}$ solution of $\mathrm{BuO}$ or AIBN in MSD (2.0 M)/n-alkanes ( $c a .50 / 50, \mathrm{v} / \mathrm{v})$ was charged into a glass ampoule. The ampoule was purged with nitrogen, sealed and immersed in a constant temperature bath regulated at $100^{\circ} \mathrm{C}$ for $10 \mathrm{~h}$. The reaction products were analyzed by GLC and GC-MS. Typical conditions of GLC analysis were as follows: column, $25 \mathrm{~m} \times 0.25 \mathrm{~mm}$ capillary column of silicon OV-1 (Shimadzu, HR-1); injector temperature, $150^{\circ} \mathrm{C}$; detector temperature, $250^{\circ} \mathrm{C}$; initial oven temperature, $50^{\circ} \mathrm{C}$; initial hold, 10 min; program rate $1,10^{\circ} \mathrm{Cmin}^{-1}$; middle oven temperature, $150^{\circ} \mathrm{C}$; middle hold, $10 \mathrm{~min}$; program rate 2 , $10^{\circ} \mathrm{C} \mathrm{min}^{-1}$; final oven temperature, $250^{\circ} \mathrm{C}$; final hold, $10 \mathrm{~min}$; carrier gas, $\mathrm{He}\left(0.4 \mathrm{ml} \mathrm{min}^{-1}\right)$; split ratio, $1: 18$.

GC retention times and MS spectra of trapping products are given below. The MS spectra showed parent peaks and characteristic fragment peaks of $117,103,91$, and 77 due to the 2-phenylallyl group of MSD.

4-Ethyl-2-phenyl-1-octene (1a). GC retention time, $35.4 \mathrm{~min}$; MS (70 eV) $\mathrm{m} / z 216\left(\mathrm{M}^{+}\right), 201,187,173,159$, $143,131,118,117,103,91,77,57,43$, and 41 .

4-Cyano-4-methyl-2-phenyl-1-pentene (1b). GC retention time, $32.3 \mathrm{~min}$; MS (70 eV) $m / z 185\left(\mathrm{M}^{+}\right), 170,158$, $143,129,117,115,103,91,77,65$, and 51.

\section{RESULTS}

The thermal decomposition of $\mathrm{BuO}$ and AIBN was carried out in MSD $(2.0 \mathrm{M}) / n$-alkanes $(c a .50 / 50, \mathrm{v} / \mathrm{v})$ at $100^{\circ} \mathrm{C}$ for $10 \mathrm{~h}$ under nitrogen. Decomposition percentages were over $99.9 \%$. In the case of $\mathrm{BuO}$, only 4-ethyl-2-phenyl-1-octene (1a) was confirmed as the trapping product derived from the acyloxy moiety. For AIBN, only 4-cyano-4-methyl-2-phenyl-1-pentene (1b) was confirmed as the trapping product. These results are consistent with the finding that aliphatic acyloxy radicals decarboxylate too fast $\left(10^{9}-10^{10} \mathrm{M}^{-1} \mathrm{~s}^{-1}\right)^{14}$ to be trapped by conventional scavengers and AIBN decomposes concertedly ${ }^{8-10}$ with the evolution of nitrogen (eq 4 and 5). Here, there was a possibility that trapping products (1) are further attacked by radicals. However, such side reaction was negligible in this study due to the presence of large amount of MSD $(2.0 \mathrm{M})$.

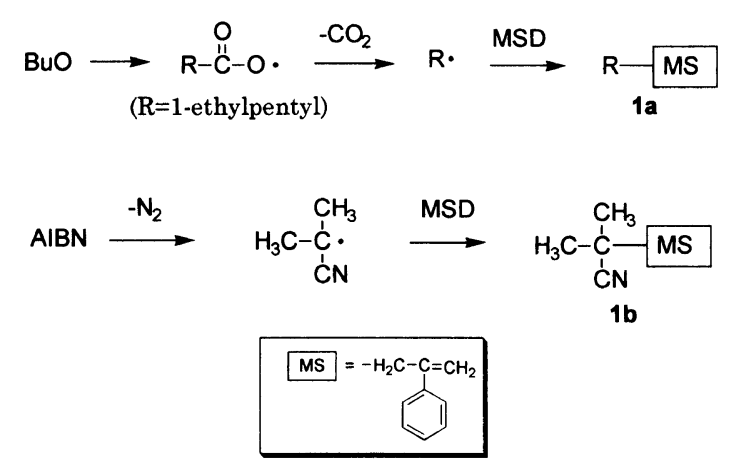

Free-radical production efficiency $(f)$ of $\mathrm{BuO}$ and AIBN was determined from the GLC yields of $1 \mathbf{a}$ and 1b. The results are shown in Table I. $f$ of $\mathrm{BuO}$ was higher than that of AIBN by $c a .0 .1-0.2 . f$ decreased with increasing carbon number of $n$-alkanes. The decrease in radical efficiency is due to the medium viscosity effect. ${ }^{11}$ The viscosity of $n$-alkanes increases with carbon number. Cage reactions between radical-radical 
Table I. Free-radical production efficiency $(f)$ of $\mathrm{BuO}$ and $\mathrm{AIBN}$ in $\mathrm{MSD} / n$-alkanes at $100^{\circ} \mathrm{C}^{\mathrm{a}}$

\begin{tabular}{lcc}
\hline \multirow{2}{*}{$n$-Alkane } & \multicolumn{2}{c}{$f$} \\
\cline { 2 - 3 } & $\mathrm{BuO}^{\mathrm{b}}$ & $\mathrm{AIBN}^{\mathrm{c}}$ \\
\hline$n$-Hexane & 0.69 & 0.53 \\
$n$-Decane & 0.60 & 0.49 \\
$n$-Dodecane & 0.57 & 0.46 \\
$n$-Heptadecane & 0.53 & 0.43 \\
\hline
\end{tabular}

${ }^{\mathrm{a}}[\mathrm{MSD}]=2.0 \mathrm{M} ; \quad \mathrm{MSD} / n$-alkanes $=c a . \quad 50 / 50 \quad(\mathrm{v} / \mathrm{v}) ; \quad[\mathrm{BuO}]=$ [AIBN] $=0.02 \mathrm{M}$. ${ }^{b} \mathrm{Mol}$ of $1 \mathrm{a} / \mathrm{mol}$ of $\mathrm{BuO} .{ }^{\mathrm{c}} \mathrm{Mol}$ of $1 \mathbf{b} / \mathrm{half} \mathrm{mol}$ of AIBN.

pairs become favorable in higher viscous medium.

\section{DISCUSSION}

Since MSD is a derivative of styrene, $f$ determined in this study should be closely related to initiator efficiency in actual styrene polymerization. We checked this by comparing our results with those reported. Recently, Yamamoto et al. ${ }^{12}$ reported the initiator efficiency of AIBN in styrene polymerization. Typically, they determined initiator efficiency by the inhibition method (measurement of induction period) in the presence of 4hydroxy-2,2,6,6-tetramethylpiperidine-1-oxyl (Tempol). They showed that the initiator efficiency depends on solvent type (aromatic or non-aromatic) and solvent viscosity. For example, the initiator efficiency in the mixture of styrene $/ n$-hexane $(50 / 50 \mathrm{v} / \mathrm{v}, \eta=0.32 \mathrm{cP})$ at $60^{\circ} \mathrm{C}$ was reported to be 0.55 . An accurate comparison must be made at the same viscosity. In Figure 1, plots of $f$ against the viscosity of the reaction medium are shown. The plots gave curves similar to the results by Yamamoto et al. ${ }^{12}$ From the curve for AIBN, $f$ at $0.32 \mathrm{cP}$ was estimated to be 0.54 . This is very close to the reported value (0.55). Thus, MSD trapping is useful for predicting initiator efficiency in styrene polymerization. Compared to the inhibition method using Tempol, MSD trapping can be carried out more conveniently and information on initiating radical species can be obtained from the trapping products.

Cage reactions of peresters are well known. Bartlett et $a l .{ }^{6}$ reported the viscosity dependence of cage reactions of $t$-butylperoxy 2-methyl-2-phenylpropionate. The percentages of cage reactions in benzene $\left(60^{\circ} \mathrm{C}, 0.39 \mathrm{cP}\right)$ and dodecane $\left(50^{\circ} \mathrm{C}, 0.94 \mathrm{cP}\right)$ were 30 and $42 \%$, indicating $f$ to be 0.70 and 0.58 , respectively. From Figure $1, f$ of $\mathrm{BuO}$ at 0.39 and $0.94 \mathrm{cP}$ was estimated as 0.67 and 0.55 , respectively. This also supports that our estimation of $f$ is made accurately.

In this study, cage reaction products were observed from the decomposition of AIBN and BuO. Decomposition in $\mathrm{MSD} / n$-dodecane gave tetramethylsuccinonitrile ( $48 \%$, based on AIBN), $t$-butyl 1-ethylpentyl ether $(17 \%$, based on $\mathrm{BuO}$ ), and 2- and 3-heptene (total $21 \%$, based on $\mathrm{BuO}$ ) for $\mathrm{AIBN}$ and $\mathrm{BuO}$, respectively. Thus, total yields of free-radical production (see Table I) and cage reactions were 94 and $95 \%$ for AIBN and $\mathrm{BuO}$, respectively, indicating excellent material balance.

$f$ of $\mathrm{BuO}$ is higher than those of AIBN by $c a .0 .1-0.2$. This clearly shows that $\mathrm{BuO}$ is a more efficient initiator

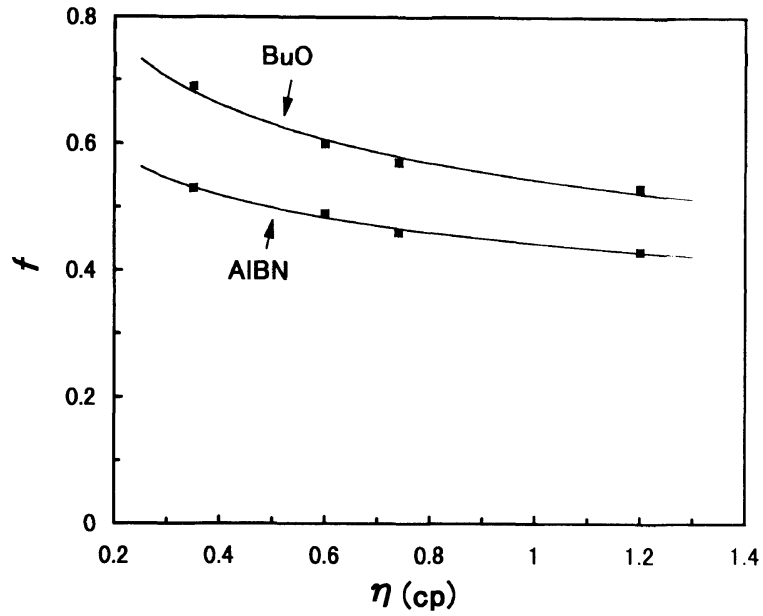

Figure 1. Plots of $f$ vs. viscosity for $\mathrm{BuO}$ and $\mathrm{AIBN}$ in $\mathrm{MSD} / n$ alkanes at $100^{\circ} \mathrm{C}$

than AIBN. Possibly, this difference is due to that in decomposition modes between AIBN and BuO. AIBN is known to decompose by a two-bond homolysis mechanism, while the decomposition mode of $\mathrm{BuO}$ is intermediate between this and a stepwise process. ${ }^{15}$ This suggests that the lifetime of cage radical pairs of AIBN (alkyl-alkyl radical pairs) is longer than that of $\mathrm{BuO}$ (alkoxy-alkyl radical pairs). Thus, the probability of AIBN undergoing cage reactions is expected to become higher than that of $\mathrm{BuO}$. For detailed discussion, however, we need more information on radical efficiency of peroxides and azo compounds.

There are a number of methods for the determination of initiator efficiency. The inhibition method above and ESR following the disappearance of added stable free radicals such as nitroxides are frequently used. These methods observe initiation radicals indirectly. MSD is not a real styrene monomer, but the present method has some advantages: (1) Free radicals which actually add to the styrenic double bond can be observed directly as trapping products. (2) Free radicals can be differentiated. (3) Addition of radicals to MSD corresponds to trapping of radicals. That is, addition and trapping can be achieved simultaneously by a single compound. (4) Trapping products are thermally stable, so reactions can be carried out even at high temperature $\left(e . g .,>100^{\circ} \mathrm{C}\right)$. (5) MSD does not have lone pairs or odd electrons that often cause abnormal decomposition of organic peroxides such as induced decomposition and ionic decomposition. (6) Trapping products are easily analyzed by conventional methods (GLC and GC-MS).

To the best of our knowledge, this paper shows for the first time the addition-fragmentation reaction useful for the determination of free-radical production efficiency of organic peroxides and azo compounds. Study on free-radical efficiency of other peroxides and azo compounds is currently underway.

\section{REFERENCES}

1. Y. Watanabe, H. Ishigaki, H. Okada, and S. Suyama, Polym. J., 29, 366 (1997).

2. Y. Watanabe, H. Ishigaki, H. Okada, and S. Suyama, Chem. 
Lett., 1089 (1993); S. Suyama, H. Ishigaki, Y. Watanabe, and T. Nakamura, Polym. J., 27, 371 (1995); S. Suyama, H. Ishigaki, Y. Watanabe, and T. Nakamura, Polym. J., 27, 503 (1995).

3. D. F. DeTar and C. Weis, J. Am. Chem. Soc., 78, 4296 (1956); D. F. DeTar and C. Weis, J. Am. Chem. Soc., 79, 3041 (1957).

4. J. W. Taylor and J. C. Martin, J. Am. Chem. Soc., 89, 6904 (1967).

5. J. E. Guillet and J. C. Gilmer, Can. J. Chem., 47, 4405 (1969).

6. F. E. Herkes, J. Friedman, and P. D. Bartlett, Intern. J. Chem. Kinetics, 1, 193 (1969).

7. R. A. Sheldon and J. K. Kochi, J. Am. Chem. Soc., 92, 4395 (1970); R. A. Sheldon and J. K. Kochi, J. Am. Chem. Soc., 92, 5175 (1970).

8. T. Koenig, in "Free Radicals," Vol. I, J. K. Kochi, Ed., John Wiley \& Sons, New York, N. Y., 1973, Chapter 3.

9. J. C. Bevington, Makromol. Chem. Macromol. Symp., 10/11, 89
(1987); J. C. Bevington, J. Polym. Mater., 10, 195 (1993).

10. D. H. Solomon and G. Moad, Makromol. Chem. Macromol. Symp., 10/11, 109 (1987).

11. F. De Schrijver and G. Smets, J. Polym. Sci., A-1, 4, 2201 (1966).

12. T. Yamamoto, J. Takeuchi, K. Konishi, S. Higashika, O. Sangen, and M. Kamachi, Polym. J., 26, 587 (1994).

13. Y. Watanabe, H. Ishigaki, H. Okada, and S. Suyama, Polym. J., 29, 603 (1997).

14. K. Fujimori, in "Organic Peroxides," W. Ando, Ed., John Wiley \& Sons, New York, N. Y., 1992, Chapter 7; J. A. Howard and J. C. Scaiano, in "Landolt-Börnstein. New Series. Radical Reaction Rates in Liquids," Vol. 13d, H. Fischer, Ed., Springer-Verlag, Berlin, 1984, p 128.

15. W. A. Pryor, E. H. Morkved, and H. T. Bickley, J. Org. Chem., 37, 1999 (1972). 